\title{
STIFF-PERSON SYNDROME FOLLOWING TICK-BORNE MENINGOENCEPHALITIS
}

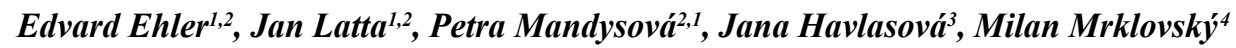 \\ Pardubice Regional Hospital, Pardubice, Czech Republic: Department of Neurology ${ }^{1}$; Faculty of Health Studies, University \\ of Pardubice, Czech Republic²; Interimun s.r.o., Policlinic HELP, Pardubice, Czech Republic ${ }^{3}$; Department of Radiodiag- \\ nostics, Multiscan s.r.o, Pardubice, Czech Republic ${ }^{4}$
}

\begin{abstract}
Summary: Stiff-person syndrome (SPS) is a rare disorder characterized by muscle stiffness and painful spasms. Misdiagnosis may occur due to the fact that the clinical picture of SPS is often atypical. The main pathophysiologic mechanism underlying the development of SPS is insufficient inhibition at the cortical and spinal levels. There is good evidence for a primary autoimmune etiology. A 61-year-old man was admitted to a neurological department due to muscle hypertonia with episodic attacks of painful spasms predominantly affecting axial muscles. The symptoms developed shortly after tickborne meningoencephalitis. Electromyography (EMG) revealed signs of continuous motor unit activity. Antibodies against glutamate decarboxylase (anti-GAD) were highly elevated. We present a case of a man who developed clinically severe anti-GAD positive SPS, provoked by tick-borne encephalitis. After therapeutic plasma exchange (TPE) a rapid, temporary improvement of the clinical and neurophysiological findings was noted. Only after being placed on long-term immunosuppression did the patient achieve stable recovery. This case supports the importance of EMG findings and demonstrates the effect of TPE as well as the need for chronic immunosuppression in severe cases of SPS.
\end{abstract}

Key words: Stiff-person syndrome; Tick-borne meningoencephalitis; Painful muscle cramps; Antibodies against glutamic acid decarboxylase (anti-GAD)

\section{Introduction}

SPS is characterized by progressive muscle hypertonia predominantly affecting axial muscles, co-contraction of antagonist muscles, and suddenly emerging episodic spasms (12). SPS, stiff limb syndrome (SLS), jerking SPS, and progressive encephalomyelitis with rigidity and myoclonus (PERM) are a family of rare, insidiously progressive diseases of the central nervous system (10). Neurophysiology has an important role in the diagnostics of SPS. Continuous motor unit activity and increased motor neuron excitability can be noted $(9,22)$. SPS is an autoimmune disorder and frequently a high amount of anti-GAD antibodies is reported. Treatment consists of pharmacological increase of inhibition (benzodiazepines, baclofen) or immunosuppressive therapy (TPE, intravenous immunoglobulin (IVIG), corticoids) (1, 19). We present a case of a man who developed anti-GAD positive SPS with progressive muscle stiffness, rigidity, and spasms following tick-borne meningoencephalitis.

\section{Case report}

A 61-year-old man was admitted to a department of neurology for muscle hypertonia (predominantly expressed in the trunk and proximal segments of his extremities) with episodic attacks of painful spasms of axial muscles and lumbago with hyperlordosis. The spasms were characterized by promptly occurring waves of muscle stiffness. The duration of the episodes was approximately 60 seconds, and 5-15 waves could be noted. The spasms and muscle hypertonia spread to his upper extremities. Cranial dislocation of the humerus in the patient's left shoulder developed due to a strong attack of spasms. During the attacks, the patient developed dyspnea, sweating, and mydriasis. Tachycardia was not always present during these painful spasms with some autonomic signs. Between attacks, the muscles were stiff, and movements were restricted. Attacks were present also during the night, and the patient suffered from insomnia. At times even his upper extremities and neck muscles were involved, more so on the left side. Hypertonia of the facial muscles was observed but without trismus. Eating difficulties were severe because of the muscle stiffness and restriction of movements in the patient's arm. There was only mild dysphagia. Constipation and urinary incontinence developed and a permanent urine catheter had to be inserted. Severe sweating (of the face, trunk, and extremities) and mydriasis occurred even between episodes of muscle spasms. The patient was not able to sit or stand mainly because of his painful spasms and hypertonia.

The patient was right-handed and was treated for a first-degree atrioventricular block, arterial hypertension, hyperuricemia, and diabetes mellitus type 2 . In the begin- 


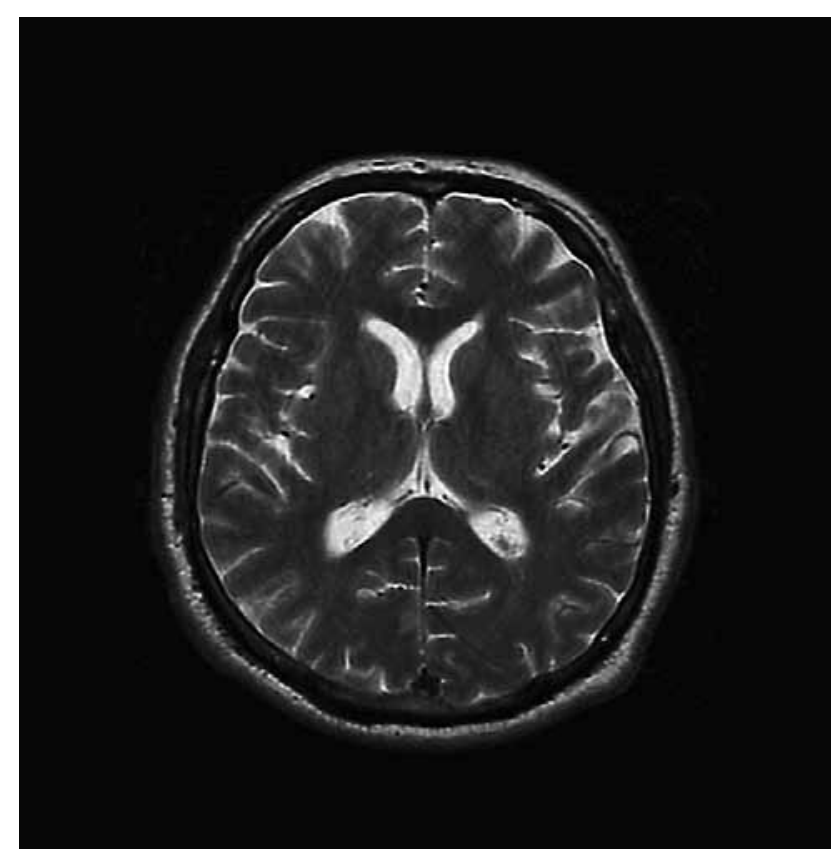

Fig. 1: Axial brain T2-weigheted MRI: normal finding

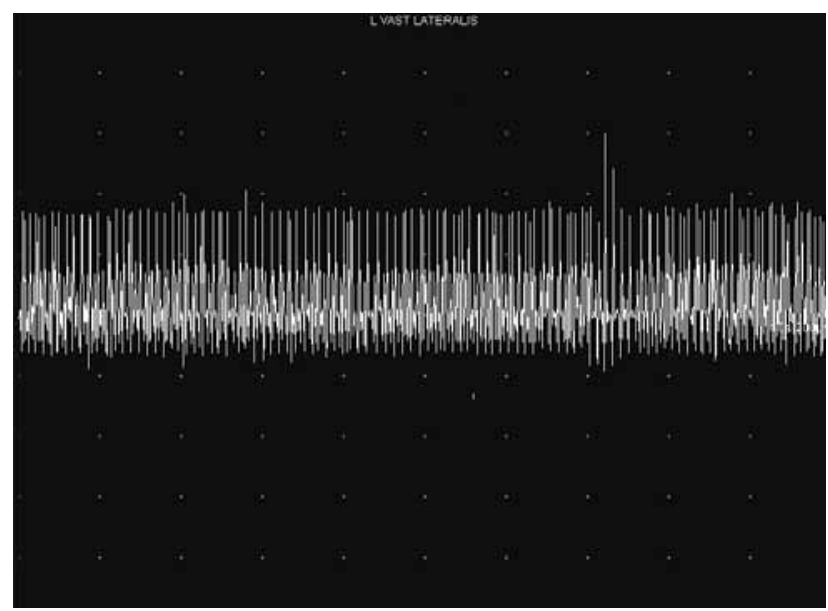

Fig. 2: Needle EMG from left vastus lateralis muscle showing continuous motor activity at rest

ning of September, he found a tick on his right thigh. Three weeks later, he developed fever, arthralgia, and headache without exanthema. For one month, he was treated for these complaints by a general practitioner and then was admitted to a department of infectious diseases (06.11.2007). Cerebrospinal fluid (CSF) pleocytosis (lymphocytes 272/3, erythrocytes 64/3) was found. Protein was elevated 0.872 (0.1-0.4 g/l) and chloride was 119 (120-130). Antibodies (type $\mathrm{IgM}$ ) against tick-borne meningoencephalitis in serum and CSF were positive (ELISA, 09.11.2007). Antibodies against Borrelia in blood and cerebrospinal fluid were negative (ELISA). Several days later (15.11.2007),
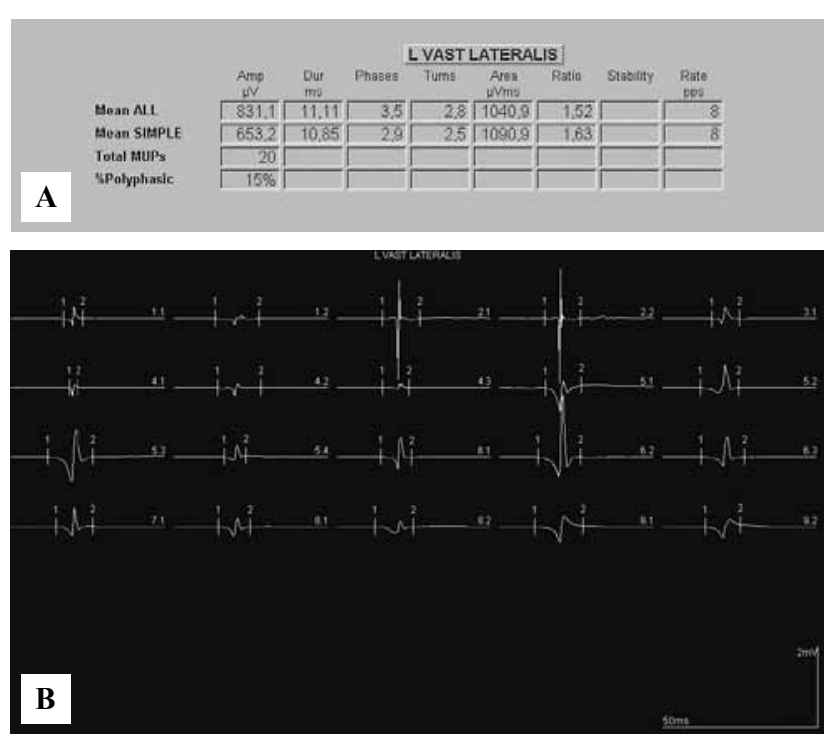

Fig. 3: Left vastus lateralis muscle. A: Results of motor potential analysis using MultiMUP programme. Normal duration, amplitude and shape of potentials; B: All 20 motor unit potentials analysed

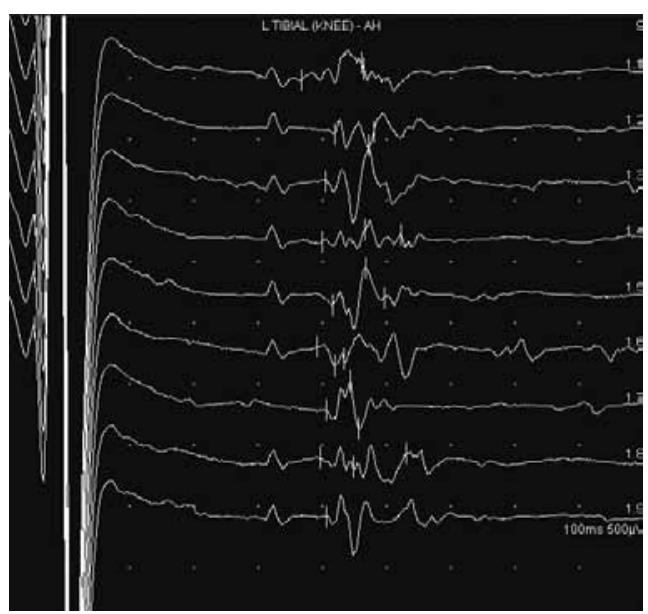

Fig. 4: Left tibial nerve - F-wave study. F-waves are polyfasic with slightly increased amplitude

muscle hypertonia and episodes of painful spasms appeared and he was unable to stand and later also to sit. He was transferred to the department of neurology (03.12.2007). Magnetic resonance imaging (MRI) of his brain was normal, and MRI of the spinal column revealed only a mild canal stenosis at L4-5 (Figure 1). Electroencephalography (EEG) did not show any abnormalities. Creatine kinase (CK) $28(0.35-3.58)$, alanin transaminase (ALT) $0.97(0.1-0.78)$ and aspartate transaminase (AST) $1.31(0.05-0.72)$ were elevated. Anti-GAD antibodies were highly elevated $>10,000$ (0-10). Other performed laboratory tests were normal. EMG showed continuous motor unit activity (normal MUPs) of 
the trunk, arm, and thigh muscles without fibrillations or fasciculations, slightly higher and polyphasic F-waves, and otherwise normal motor and sensory neurography. (Figure 2, 3, 4)

The patient was treated with an increasing dose of diazepam (up to $100 \mathrm{mg} /$ day) and baclofen (up to $75 \mathrm{mg} /$ day). TPE was performed in 5 sessions with approximately 14.5 liters of plasma exchanged. After the TPE and after oral treatment, the patient stabilized; he was able to sit and stand as well as to perform a few steps. But 2 months later (26.03.2008), muscle hypertonia with painful spasms of his muscles relapsed. He responded again to TPE. At the same time, he was placed on immunosuppressive therapy with dexamethasone $(4 \mathrm{mg})$ and azathioprine $(100 \mathrm{mg})$. After 5 months, we could gradually reduce and then completely stop diazepam and baclofen (15.07.2010). Currently, the patient's stiffness is mild, without painful spasms, and he is able to walk independently.

\section{Discussion}

SPS is a rare, insidiously progressive disease of the central nervous system. It is characterized by rigidity, particularly of axial muscles with superimposed stimulussensitive spasms (10). Painful spasms are provoked by movements, tactile or acoustic stimulation or simply by emotions. They are not provoked by visual stimulation. The spasms are associated with intense pain and can sometimes persist for days. Spasms can affect facial muscles and the larynx, causing stridor, and occasionally can be so severe in the limbs that they can cause fractures $(1,10)$. In our patient, SPS developed quickly. After several days, he was unable to stand or sit, and he had severe muscle stiffness restricting movements as well as long periods of cumulative attacks of painful spasms with hyperlordosis. He suffered a left shoulder dislocation during a very strong spasm attack. Painful spasms were mostly in the trunk and abdomen. Breathing was difficult, but hypoxemia did not develop.

SPS can be mistaken for psychogenic disorders and many other diseases characterized by muscle stiffness, painful spasms, anxiety, and autonomic signs $(12,14)$. Diagnostic criteria (Table 1) consist of core, supplementary, and associated features (10). At the beginning of the diagnostic process, tetanus, neuromyotonia, progressive encephalomyelitis with rigidity and myoclonus and other diseases associated with muscle stiffness and spasms should be differentiated (8). The patient's symptoms, clinical signs, EMG (continuous motor unit activity with normal MUPs), and high anti-GAD65 level were all parameters favoring the diagnosis of SPS (18).

The role of electrophysiology in SPS is complex. Routine motor and sensory nerve conduction studies are normal (12). Needle EMG of symptomatic muscles (axial, hip and the shoulder girdle) reveals spontaneous discharges of

Tab. 1: Diagnostic criteria of stiff-person syndrome

\begin{tabular}{|l|l|}
\hline \multirow{5}{*}{ Core diagnostic criteria } & A) Positive \\
& Stiffess and rigidity of axial muscles \\
& Abnormal axial posture (90\% lumbar hyperlordosis) \\
& Stimulus-sensitive spasms (movement, emotional, auditory) \\
& EMG evidence of continuous muscle unit activity \\
\cline { 2 - 3 } & B) Negative \\
& Absent brainstem, pyramidal, extrapyramidal, lower motor neuron signs \\
& No sphincter disturbance \\
& No sensory disturbance \\
& Absence of chronic pain syndrome \\
& No cognitive impairment \\
\hline \multirow{5}{*}{ Supplementary diagnostic criteria } & Stiffness and rigidity in proximal limb muscles \\
& Resolution of rigidity and stiffness with intravenous benzodiazepines \\
& EMG evidence of abnormal exteroceptive reflexes \\
& Serum anti-GAD65 antibodies \\
& CSF protein level $>0.6$ g/l \\
& CSF anti-GAD antibodies \\
& Non-habituating head retraction reflex (i.e. glabellar reflex) \\
\hline \multirow{5}{*}{ Associated clinical features } & Ocular signs \\
& Paroxysmal dysautonomia (hyperpyrexia, diaphoresis, tachypnea, tachycardia, \\
& pupillomotor, hypertension) \\
& Paroxysmal fear \\
\hline
\end{tabular}

Diagnostic criteria (6) 
normal MUPs. Other waveforms (fibrillations, fasciculations, myotonic or neuromyotonic discharges), which can be observed in other diseases, are not present. In addition, electrical silence in the case of muscle hardening (contracture) suggests the presence of a metabolic muscle disease (1, 11, 21). A number of other neurophysiological techniques may demonstrate hyperexcitability of the reflex arch and motoneurons (9). Vibration-induced inhibition of H-reflex is a GABAergic phenomenon that may be suppressed in SPS (1). Transcranial magnetic stimulation reveals a silent period reduced by $20 \%(15,20)$. The blink reflex may be enhanced in SPS, leading to recruitment of muscles not typically activated, and $\mathrm{R} 2$ response is not suppressed after a conditioning stimulus $(1,10)$. Our patient's needle EMG revealed continuous motor unit activity, with normal MUPs, in the axial and proximal arm and leg muscles. Motor and sensory nerve conduction studies were normal. F-waves were characterized by $100 \%$ persistence, long duration, and a slightly higher amplitude (up to 5\% amplitude of M-response is the norm; we found up to $7.5 \%$ ). F-waves parameters supported the presence of a hyperexcitability state of the reflex arch in SPS (18).

The leading pathophysiologic moment in the development of SPS is a disorder of inhibition. The insufficient inhibition of cortical neurons and subsequently of the spinal motor reflex arch is due to the effects of anti-GAD autoantibodies leading to a lack of $\gamma$-aminobutyric acid (GABA) (19). GABA (and glycin) plays an important role in regulating neuronal excitability. The lack of GABA leads to insufficient inhibition, thus resulting in continuous motor activity. The disturbance of inhibition at the level of central descendent pathways is necessary for full development of SPS symptoms (including motor and non-motor symptoms) (1). Anti-GAD antibodies can be determined by molecular weight and by antigen properties to anti-GAD65 (65 kilodaltons), which are present in $80 \%$ of SPS cases, and to anti-GAD67, which are present in $60 \%$ of the cases (17). Our patient had a more than a 1000 times higher level of the anti-GAD antibody in the serum. After a high volume TPE, the patient's muscle stiffness, painful spasms and deformity of the spine regressed. In addition, his sweating, mydriasis, constipation, and diaphoresis improved.

As in other disorders of autoimmunity, patients with SPS appear to have an increased incidence of concomitant immune-mediated disorders both within and outside of the nervous system (1). This is particularly true in those who have GAD antibodies. The prevalence of these comorbidities may be as high as $80 \%$. Immune-mediated neurologic diseases associated with SPS are encephalomyelitis, cerebellar syndromes, myasthenia gravis, and sensory neuropathy (17). Systemic diseases associated with SPS include thyreopathies, celiac disease, pernicious anemia, rheumatoid arthritis, systemic lupus erythematosus, and vitiligo (10). Diabetes mellitus is particularly prevalent and may exist in up to $70 \%$ of patients with SPS. The initiation of SPS development is commonly associated with a broad range of immune factors (intercurrent virosis, neuroinfection - Lyme borreliosis, West Nile fever), and it can be associated with muscle hypertonia as well $(12,16)$. In one recently described case, the disease had a clear temporal link to a West Nile virus infection (13). Sequence homology between GAD65 and West Nile virus raises the possibility of molecular mimicry. Sequence analysis also reveals candidate homologous regions in coxsackievirus and cytomegalovirus (10). Our patient had type 2 diabetes mellitus and the onset of his symptoms was associated with prolonged fever and polyarthralgia after tick-borne meningoencephalitis.

Frequently, SPS is difficult to diagnose due to several reasons. It is not a well-known neurological disorder. Clinical presentation of SPS can vary from one case to another even if the typical symptoms are mostly present - muscle stiffness, painful spasms coming in waves, sweating, difficult gait, and restriction of movements. In the beginning, the condition may be misdiagnosed, usually as vertebrogenic pain syndrome (7), dystonia or orthostatic tremor (11), encephalomyelitis, generalized myoclonus (8), or Isaac's syndrome (18). Because of the presence of painful generalized spasms, Clostridium tetani infection must be considered as well, and the skin should be checked for wounds. Trismus usually does not occur in SPS. Another reason for SPS misdiagnosis or delayed diagnosis is the occurrence of localized forms of SPS (stiff-limb syndrome) or the occurrence of disease forms associated with other symptoms (SPS plus syndromes) $(5,6)$. The clinical approach to these disorders necessitates a precise description of the character of the stiffness and spasms as well as a description of triggers such as tactile stimulation and emotional stimuli. A response to benzodiazepines and baclofen may aid in the diagnosis (2). Vegetative symptoms like sweating with a distribution within a certain area and mydriasis are obligatory and thus important, while sphincter disorders have not been described so frequently (5). Our patient had severe rigidity with painful spasms, autonomic signs, and anxiety, and because of pain and spasms in the lumbar region, he was not able to sit or even to stand up. This was a typical observation described in the literature $(12,18)$.

Treatment of SPS involves the use of both symptomatic agents to enhance GABAergic influences and immunomodulating therapy aimed at suppressing unwanted immune-mediated effects. Rigidity and spasms usually respond to GABA agonists such as benzodiazepines or baclofen (1). In addition, gabapentin and pregabalin are frequently used. In mild and some moderate cases, this treatment is sufficient, especially in patients with an associated generalized anxiety. Pain crises can be managed with opioids (10). Patients with muscle stiffness and spasms resistant to benzodiazepines can benefit from treatment with, intravenous propofol infusion, or intrathecal baclofen. Intramuscular injection of botulinum toxin may be useful to treat severe rigidity. However, botulinum toxin treatment is limited by its symptomatic effect and by the need for large doses to adequately address large axial muscle groups. 
Patient who continue to progress should be considered for disease-modifying therapy with immunomodulatory or immunosuppressive agents (12). Steroids, cyclophosphamide, azathioprine, mycophenolate mofetil, and plasma exchange have all been used with varying success. The only evidence-based immunomodulating therapy is intravenous immunoglobulin, given regularly (one controlled clinical trial) (8). Recently reported treatment with rituximab induced a lasting clinical repision $(3,4)$. Our patient was treated by increasing doses of benzodiazepines and baclofen. Because of only partial effects, we opted for plasma exchange. Immediately after the TPE, the patient's painful spasms and rigidity (to a lesser extent) decreased. The symptomatic therapy was reduced and the patient was able to walk (only several steps, indoors). After 2 months, all the spasms, autonomic signs, and stiffness returned. We used a second round of plasma exchange and initiated chronic immunosuppression (dexamethasone, azathioprine $100 \mathrm{mg} /$ day). Five months later, the benzodiazepines and baclofen were stopped and now he can walk up to $5 \mathrm{~km}$ without support. In severe cases, we strongly recommend that chronic oral immunosuppression be initiated early.

\section{Conclusion}

SPS is characterized by fluctuating muscle stiffness spreading from the axial muscles to the proximal segments of limbs and by attacks of painful spasms. The leading pathophysiological moment is insufficient inhibition of the GABAergic pathways. There is a broad range of immune factors, able to trigger SPS. In our case, we have concluded that tick-borne encephalitis could have been a trigger of immunological changes leading to SPS. TPE was able to promptly induce remission in anti-GAD positive SPS. Subsequent chronic immunosuppressive treatment was necessary to achieve long-term improvement.

\section{References}

1. Amato AA, Russel JA. Neuromuscular disorders. New York: McGraw Hill Medical 2008: $776 \mathrm{~s}$.

2. Andreadou E, Katoutlas E, Stagos C, Vassilopoulos D. Stiff person syndrome: avoiding misdiagnosis. Neurol Sciences 2007; 28: 35-38.

3. Bacorro EA, Tehrani R. Stiff-person syndrome: persistent elevation of glutamic acid decarboxylase antibodies despite successful treatment with rituximab. J Clin Rheumatol 2010; 16: 237-9.

4. Baker MR, Das M, Isaacs J, Fawcett PR, Bates D. Treatment of stiff person syndrome with rituximab. J Neurol Neurosurg Psychiatry 2005; 76: 999-1001.

5. Barker RA, Revesz T, Tom M, Marsden CD, Brown P. Review of 23 patients affected by the stiff man syndrome: clinical subdivision into stiff trunk (man) syndrome, stiff limb syndrome, and progressive encephalomyelitis with rigidity. J Neurol Neurosurg Psychiatry 1998; 65: 633-640.

6. Bartsch T, Herzog J, Baron R, Neuschl G. The stiff limb syndrome - a new case and a literature review. J Neurol 2003; 250: 488-490.

7. Bastin A, Gurmin V, Mediwake R, Gibbs J, Beynon H. Stiff man syndrome presenting with low back pain. Ann Rheum Dis 2002; 61: 939-940.

8. Brown P, Marsden CD. The stiff man and stiff man plus syndromes. J Neurol 1999; 246: 648-652.

9. Burns TM. A step forward for stiff-person syndrome. The Lancet 2005; 365 : $1365-1367$.

10. Duddy ME, Baker MR. Stiff Person syndrome. In: Pourmand R. Immune-mediated neuromuscular diseases. Basel: Karger 2009: 147-165.

11. Floeter MK, Valls-Solé J, Toro C, Jacobowitz D, Hallett M. Physiologic studies of spinal inhibitory circuits in patients with stiff-person syndrome. Neurology 1998 51: 85-93.

12. Goetz ChG (ed). Textbook of clinical neurology. Philadelphia: Saunders 2003 1306.

13. Hassin-Baer S, Kordon ED, Hulman L, et al. Stiff-Person Syndrome Following West Nile Fever. Arch Neurol. 2004; 61(6): 938-941.

14. Kiriakos CR, Franco KN. Stiff-Man Syndrome: A Case Report and Review of the Literature. Psychosomatics 2002; 43: 243-244.

15. Logullo F, Di Bella P, Provniciali L. Motor evoked potentials in a case of stiff-man syndrome: a longitudinal study. The Ital J Neuro Sci 1999; 20:133-138.

16. Martin R, Meinck HM, Schulte-Mattler W, Ricker K and Mertens HG. Borrelia burgdorferi myelitis presenting as a partial stiff man syndrome. J Neurol 1990; 237: $51-54$.

17. Meinck, H-M, Faber, L, Morgenthaler, N, et al. Antibodies against glutamic acid decarboxylase: prevalence in neurological diseases. J Neurol Neurosurg Psychiatry 2001; 71: 100-103.

18. Oh SJ. (Ed.) Principles of clinical electromyography. Case studies. Baltimore: Williams Wilkins 1998, 604.

19. Raju R, Rakocevic G, Chen Z, et al. Autoimmunity to $\mathrm{GABA}_{\mathrm{A}}$-receptor-associated protein in stiff-person syndrome. Brain 2006; 129: 3270-3276.

20. Sandbrink F, Syed NA, Fujii MD, Dalakas MC, Floeter MK. Motor cortex excitabillity in stiff-person syndrome. Brain 2000; 123: 2231-2239.

21. Valls-Solé J, Montero J. Role of EMG evaluation in muscle hyperactivity syndromes. J Neurol 2004; 251: 251-260.

22. Vasconcelos OM, Marinos CD. Stiff-person syndrome. Current treatment opinions in Neurology 2003: 79-90.

Received: 21/03/2011

\section{Corresponding author:}

Accepted in revised form: 04/11/2011

doc. MUDr. Edvard Ehler, CSc. Department of Neurology, Pardubice Regional Hospital, Kyjevská 44, Pardubice, 532 03; e-mail: edvard.ehler@nemocnice-pardubice.cz 